\title{
СТАБИЛИЗАЦИЯ СИСТЕМЫ СВЯЗАННЫХ ОБРАТНЫХ МАЯТНИКОВ ВЕРТИКАЛЬНЫМИ ОСЦИЛЛЯЦИЯМИ
}

\author{
М. Е. Семёнов ${ }^{\star, * *, * * *}$, М. А. Попов ${ }^{\star}$ \\ *Федеральный исследовательский иентр «Единая геофизическая служба \\ Российской академии наук» (2. Обнинск), \\ **Воронежский государственный университет \\ ***Воронежский государственный технический университет
}

Поступила в редакцию 29.09.2018 г.

\begin{abstract}
Аннотация. В статье рассматривается математическая модель, описывающая динамику двух обратных маятников с упругой связью (пружиной). Предложено программное управление движением системы, осуществляемое посредством вертикальных осцилляций общей нижней точки крепления маятников. Проведено исследование динамики указанной механической системы, сформулированы условия, обеспечивающие ее стабилизацию. Построены зоны устойчивости в пространствах исходных и безразмерных параметров. Представлены эволюции зон устойчивости в зависимости от значений жёсткости пружины. В работе также приведены результаты численных экспериментов, иллюстрирующих динамику системы.

Ключевые слова: обратный маятник, связанные осцилляторы, стабилизация, управление, зоны устойчивости.

Annotation. In this paper we propose a mathematical model consisting of two inverse pendulums with an elastic coupling (by spring). We propose a dynamic programmed control of the model motion, implemented through vertical oscillations of the common pendulums pivot point. We investigate dynamics of this mechanical system, and formulated a condition for identifying stability of the system. We constructed stability zones in the spaces of the original and dimensionless parameters. Also, we obtain evolution of stability zones depending on spring stiffness values. In conclusion, we presented results of numerical software experiments for various system configurations.
\end{abstract}

Keywords: inverted pendulum, linked oscillators, stabilization, controlling, stability zones.

\section{ВВЕДЕНИЕ}

Теория колебаний нелинейных систем широко применяется при моделировании различных физических процессов и явлений [1], таких как колебания в электрических цепях, состоящих из нескольких взаимосвязанных контуров, молекул в жидкостях и твердых телах и т. д. В таких системах реализуются разнообразные дисперсионные зависимости, на основе которых исследуется распространение волн в нелинейных средах. Большинство таких моделей систем основывается на законах движения простейших связанных осцилляторов и их цепочек, динамика кото-

(C) Семёнов М. Е., Попов М. А., 2018 рых формализуется посредством линейных и нелинейных уравнений. Во множестве подобных задач рассматриваются колебания маятников с устойчивым нулевым положением [2-4]. Подробный обзор последних результатов в этой области приведён, например в [5].

В то же время, в ряде практически важных задач (например, проблема стабилизации плазмы, колебания атомов в телах с большой температурой) нулевое положение является неустойчивым. В связи с этим отметим классическую задачу стабилизации верхнего положения обратного маятника[6]. При решении этой задачи основное внимание уделялось проблеме стабилизации неустойчивого положения равновесия маятника путем движений нижней точки крепления. Этой 
проблеме посвящено огромное количество публикаций, достаточно подробный обзор которых содержится в [7]. Отметим, что стабилизация верхнего положения может быть достигнута различными способами: периодическими вертикальными осцилляциями нижней точки крепления, с использованием принципа обратной связи и др. Задача стабилизации маятника с помощью вертикальных осцилляций точки крепления хорошо изучена. Теоретическое объяснение этого явления было сделано Стефенсоном в 1908 году [8]. Физическое же объяснение динамической стабилизации перевернутого маятника вертикальными осцилляциями точки крепления было предложено академиком П.Л. Капицей в 1951 году, который также дал и оценку снизу частоты вибрации, при которой верхнее положение становится устойчивым [9].

Согласно $[9,10]$, уравнение динамики маятника единичной массы имеет вид:

$$
\ddot{\varphi}-\frac{1}{l}(g+\ddot{f}(t)) \sin \varphi=0,
$$

где $\varphi$ - угол отклонения маятника от вертикали, $l$ - длина маятника, $g$ - ускорение свободного падения, $f(t)$ - закон движения крепления.

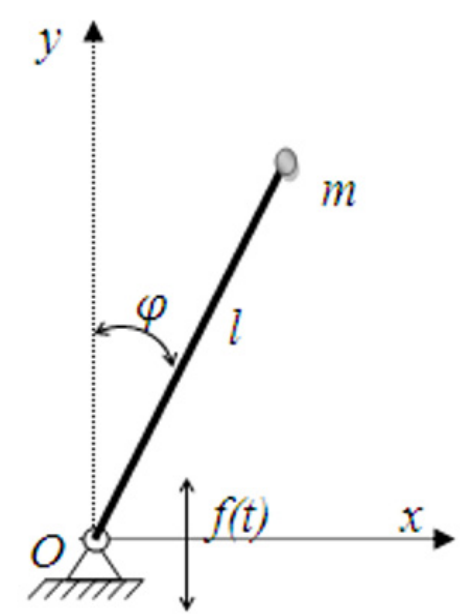

Рис. 1. Модель вертикального маятника с осииллирующим креплением

При движении нижней точки крепления по гармоническому закону уравнение (1) сводится к хорошо известному уравнению Матьё [4]. Для адекватного описания динамики реальных физических и механических систем необходимо учитывать эффекты гистерезис- ной природы - люфты, упоры $[11,12]$. Исследование устойчивости таких систем детально проведены в [13-17].

Отметим, что проблеме стабилизации систем связанных неустойчивых осцилляторов посвящено совсем немного работ. Исследование возможности стабилизации таких систем при помощи горизонтальных движений проведено в [18-20]. Также представляет интерес другая задача - возможность управления с помощью вертикальных осцилляций точки крепления.

В настоящей работе исследуется система, состоящая из двух перевернутых маятников, соединённых пружиной, с периодическим управляющим воздействием.

\section{ПОСТАНОВКА ЗАДАЧИ}

Ниже рассматривается система, в которой основания обоих маятников находятся на общей планке, которая может перемещаться вдоль вертикальной оси. Физическая модель изучаемой системы приведена на рис. 2.

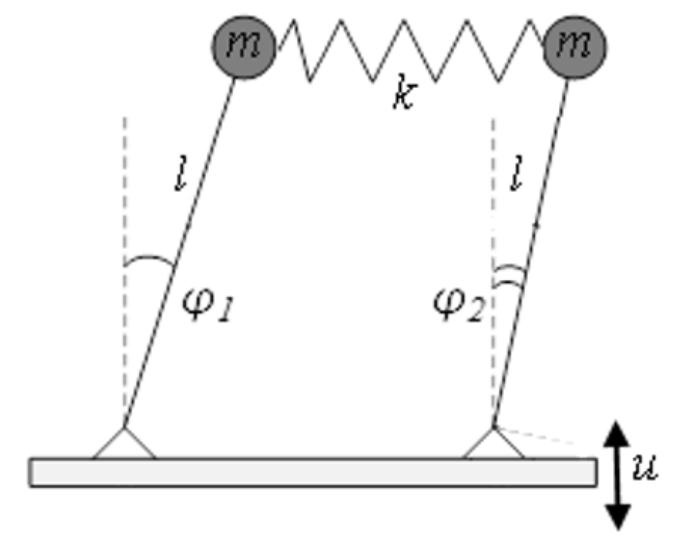

Рис. 2. Физическая модель связанных маятников

При этом отметим, что при жёсткости пружины $k=0$, получается система из двух одинаковых маятников с общим управлением. В таком случае движение каждого из маятников описывается уравнением Матьё. В случае $k \rightarrow \infty$, связь становится жёсткой (пружину можно заменить нерастяжимым стержнем) и оба маятника движутся как один. Таким образом, в обоих случаях для стабилизации системы достаточно воспользоваться известными результатами $[9,10]$. 
Ниже рассматривается случай, когда жёсткость пружины $k \in(0 ; \infty)$. Будем считать, что планка осуществляет движение так, что её ускорение периодически с частотой $\omega$, а амплитуда колебаний равна $A \omega^{2}-a \omega^{2}$. Это соответствует тому, что линеаризованное уравнение движения описанной выше системы будет иметь вид:

$$
\left\{\begin{array}{c}
\ddot{\varphi}_{1}-\frac{1}{l}\left(g+A \omega^{2} w(t)\right) \varphi_{1}+\frac{k l^{2}}{J}\left(\varphi_{2}-\varphi_{1}\right)=0 \\
\ddot{\varphi}_{2}-\frac{1}{l}\left(g+A \omega^{2} w(t)\right) \varphi_{2}-\frac{k l^{2}}{J}\left(\varphi_{2}-\varphi_{1}\right)=0 \\
w(t)=-\operatorname{sign}(\sin (\omega t)), \\
\varphi_{1}(0)=\varphi_{11}, \quad \dot{\varphi}_{1}(0)=\varphi_{12}, \\
\varphi_{2}(0)=\varphi_{21}, \quad \dot{\varphi}_{2}(0)=\varphi_{22},
\end{array}\right.
$$

где $w(t)$ - ускорение основания маятников, $J$ - момент инерции маятника, $k$ - жёсткость пружины.

Таким образом, задача заключается в следующем:

1) Выбор такого режима управления, который бы обеспечивал стабилизацию системы.

2) Определение зон устойчивости в пространстве параметров (амплитуда-частота), обеспечивающих стабилизацию.

\section{ОСНОВНЫЕ УРАВНЕНИЯ ДИНАМИКИ}

Перейдем к безразмерным единицам, сделав в (2) следующую замену:

$$
\tau=\omega t, \alpha=\frac{g}{l \omega^{2}}, \beta=\frac{A}{l}, \gamma=\frac{k l^{2}}{J} .
$$

В результате получим следующую систему:

$$
\begin{gathered}
\left\{\begin{array}{c}
\ddot{\varphi}_{1}-(\alpha+\beta \operatorname{sign}(\sin \tau)) \varphi_{1}+\gamma\left(\varphi_{2}-\varphi_{1}\right)=0 \\
\ddot{\varphi}_{2}-(\alpha+\beta \operatorname{sign}(\sin \tau)) \varphi_{2}-\gamma\left(\varphi_{2}-\varphi_{1}\right)=0
\end{array}\right. \\
\varphi_{1}(0)=\varphi_{11}, \dot{\varphi}_{1}(0)=\varphi_{12}, \\
\varphi_{2}(0)=\varphi_{21}, \dot{\varphi}_{2}(0)=\varphi_{22},
\end{gathered}
$$

Запишем (4) в виде эквивалентной системы

$$
\begin{gathered}
\left\{\begin{array}{l}
\dot{z}_{1}=z_{2} \\
\dot{z}_{2}=p(\tau) z_{1}+\gamma z_{3} \\
\dot{z}_{3}=z_{4} \\
\dot{z}_{4}=p(\tau) z_{3}+\gamma z_{1}
\end{array}\right. \\
\varphi_{1}(0)=\varphi_{11}, \dot{\varphi}_{1}(0)=\varphi_{12},
\end{gathered}
$$

$$
\varphi_{2}(0)=\varphi_{21}, \dot{\varphi}_{2}(0)=\varphi_{22},
$$

где $p(\tau)=\alpha+\beta \operatorname{sign}(\sin \tau)-\gamma$. Матрица указанной системы имеет следующий вид:

$$
\mathbf{P}(\tau)=\left(\begin{array}{cccc}
0 & 1 & 0 & 0 \\
p(\tau) & 0 & \gamma & 0 \\
0 & 0 & 0 & 1 \\
\gamma & 0 & p(\tau) & 0
\end{array}\right),
$$

В силу сделанных предположений матрица $\mathbf{P}(\tau)$, является периодической функцией времени с периодом $2 \pi$, так что для любого момента времени $\tau$ справедливо равенство $\mathbf{P}(\tau+2 \pi) \equiv \mathbf{P}(\tau)$.

Будем говорить, что уравнение (4) устойчиво или неустойчиво по Лагранжу, если устойчива или, соответственно, неустойчива система (5). То есть все решения $z(\tau)$ ограничены на $[0, \infty)$. В силу периодичности матрицы системы из результатов Флоке [13] следует, что задача исследования устойчивости сводится к нахождению фундаментальной матрицы решений в момент $2 \pi$ - матрицы монодромии и оценке ее собственных значений (мультипликаторов). Для устойчивости системы необходимо и достаточно, чтобы все мультипликаторы находились внутри единичного круга:

$$
|\lambda|<1
$$

В силу того, что матрица $\mathbf{P}(\tau)$ кусочно-постоянная, то фундаментальная система решений и, следовательно, матрица монодромии могут быть представлены в явном виде. Для этого рассмотрим поведение кусочно-постоянной функции $r(\tau)=\operatorname{sign}(\sin \tau)$ с периодом $2 \pi$ и соответственно $p(\tau)$ (рис.3).

Из рис. 3 видно, что система (4) на промежутке $(0,2 \pi)$ может быть описана 2-мя линейными системами с постоянными коэффициентами:

$$
\left\{\begin{array}{l}
\dot{z}_{1}^{1}=z_{2}^{1} \\
\dot{z}_{2}^{1}=(\alpha+\beta-\gamma) z_{1}^{1}+\gamma z_{3}^{1} \\
\dot{z}_{3}^{1}=z_{4}^{1} \\
\dot{z}_{4}^{1}=(\alpha+\beta-\gamma) z_{3}^{1}+\gamma z_{1}^{1}
\end{array} \quad \tau \in[0, \pi]\right.
$$



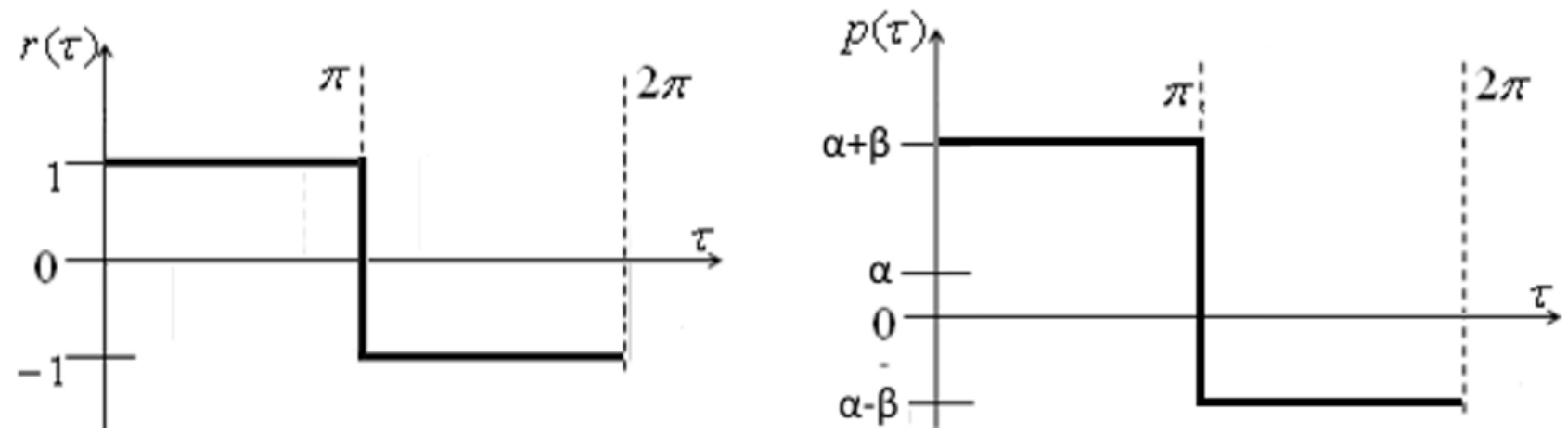

Рис. 3. Графики функиий $r(\tau)$ и $p(\tau)$

$$
\left\{\begin{array}{l}
\dot{z}_{1}^{1}=z_{2}^{1} \\
\dot{z}_{2}^{1}=(\alpha-\beta-\gamma) z_{1}^{1}+\gamma z_{3}^{1} \\
\dot{z}_{3}^{1}=z_{4}^{1} \\
\dot{z}_{4}^{1}=(\alpha-\beta-\gamma) z_{3}^{1}+\gamma z_{1}^{1}
\end{array} \quad \tau \in[\pi, 2 \pi]\right.
$$

Так как фундаментальная матрица должна быть непрерывна, то решения систем (7)-(8) должны совпадать в момент времени $\pi$ :

$$
Z^{1}(0)=\mathbf{E}, Z^{1}(\pi)=Z^{2}(\pi),
$$

где $\mathbf{E}$ - единичная матрица. Последовательно интегрируя системы (7)-(8), найдём:

$$
\begin{gathered}
Z^{1}(\tau)=\frac{1}{2}\left(\begin{array}{cc}
Z_{1,1}^{1} & Z_{1,2}^{1} \\
Z_{2,1}^{1} & Z_{2,2}^{1}
\end{array}\right), \\
Z^{2}(\tau)=Z^{1}(\pi) \frac{1}{2}\left(\begin{array}{cc}
Z_{1,1}^{2} & Z_{1,2}^{2} \\
Z_{2,1}^{2} & Z_{2,2}^{2}
\end{array}\right),
\end{gathered}
$$

где $Z_{1,1}^{1}=\left(\begin{array}{cc}\operatorname{ch}(a \tau) & \operatorname{ch}(b \tau) \\ a \operatorname{sh}(a \tau) & b \operatorname{sh}(b \tau)\end{array}\right)$,

$$
\begin{aligned}
& Z_{1,2}^{1}=\left(\begin{array}{ll}
\frac{1}{a} \operatorname{sh}(a \tau) & \frac{1}{b} \operatorname{sh}(b \tau) \\
\operatorname{ch}(a \tau) & \operatorname{ch}(b \tau)
\end{array}\right), \\
& Z_{2,1}^{1}=\left(\begin{array}{cc}
-\operatorname{ch}(a \tau) & \operatorname{ch}(b \tau) \\
-a \operatorname{sh}(a \tau) & b \operatorname{sh}(b \tau)
\end{array}\right), \\
& Z_{2,2}^{1}=\left(\begin{array}{cc}
-\frac{1}{a} \operatorname{sh}(a \tau) & \frac{1}{b} \operatorname{sh}(b \tau) \\
-\operatorname{ch}(a \tau) & \operatorname{ch}(b \tau)
\end{array}\right), \\
& Z_{1,1}^{2}=\left(\begin{array}{cc}
\operatorname{ch}(c(\tau-\pi)) & \operatorname{ch}(d(\tau-\pi)) \\
c \operatorname{sh}(c(\tau-\pi)) & d \operatorname{sh}(d(\tau-\pi))
\end{array}\right), \\
& Z_{1,2}^{2}=\left(\begin{array}{ll}
\frac{1}{c} \operatorname{sh}(c(\tau-\pi)) & \frac{1}{d} \operatorname{sh}(d(\tau-\pi)) \\
\operatorname{ch}(c(\tau-\pi)) & \operatorname{ch}(d(\tau-\pi))
\end{array}\right),
\end{aligned}
$$

$$
\begin{aligned}
& Z_{2,1}^{2}=\left(\begin{array}{cc}
-\operatorname{ch}(c(\tau-\pi)) & \operatorname{ch}(d(\tau-\pi)) \\
-c \operatorname{sh}(c(\tau-\pi)) & d \operatorname{sh}(d(\tau-\pi))
\end{array}\right), \\
& Z_{2,2}^{2}=\left(\begin{array}{cc}
-\frac{1}{c} \operatorname{sh}(c(\tau-\pi)) & \frac{1}{d} \operatorname{sh}(d(\tau-\pi)) \\
-\operatorname{ch}(c(\tau-\pi)) & \operatorname{ch}(d(\tau-\pi))
\end{array}\right),
\end{aligned}
$$

где $a=\sqrt{\alpha+\beta}, b=\sqrt{\alpha+\beta-2 \gamma}$ и $c=\sqrt{\alpha-\beta}$, $d=\sqrt{\alpha-\beta-2 \gamma}$.

Тогда, полагая в $Z^{2}(\tau) \tau=2 \pi$, получим матрицу монодромии системы (4) в следующем виде:

$$
H=Z(2 \pi)=\frac{1}{4}\left(\begin{array}{ll}
Z_{1,1}^{1} & Z_{1,2}^{1} \\
Z_{2,1}^{1} & Z_{2,2}^{1}
\end{array}\right)\left(\begin{array}{cc}
Z_{1,1}^{2} & Z_{1,2}^{2} \\
Z_{2,1}^{2} & Z_{2,2}^{2}
\end{array}\right) .
$$

Характеристическое уравнение для матрицы $Z(2 \pi)$ имеет вид:

$$
\begin{aligned}
\operatorname{det}(H-\lambda \mathbf{E}) & =\left|\begin{array}{cccc}
h_{11}-\lambda & h_{12} & h_{13} & h_{14} \\
h_{21} & h_{22}-\lambda & h_{23} & h_{24} \\
h_{31} & h_{32} & h_{33}-\lambda & h_{34} \\
h_{41} & h_{42} & h_{43} & h_{44}-\lambda
\end{array}\right|= \\
& =\lambda^{4}+q_{1} \lambda^{3}+q_{2} \lambda^{2}+q_{3} \lambda+q_{4}=0, \\
\text { где } q_{4} & =(-1)^{4} e^{\int^{T} S p(\mathbf{P}(\tau)) d \tau}=1[14] .
\end{aligned}
$$

Для идентификации зон устойчивости в пространстве параметров можно воспользоваться критерием Рауса - Гурвица. Для этого нужно сделать замену $\lambda=\frac{1+\mu}{1-\mu}$ (это отображение сопоставляет единичному кругу левую часть комплексной плоскости), получив уравнение с комплексными коэффициентами вида $u_{0} \mu^{4}+u_{1} \mu^{3}+u_{2} \mu^{2}+u_{3} \mu+u_{4}=0$. Для того, чтобы корни соответствующего уравнения лежали в левой части комплексной плоскости, а как следствие, корни уравнения (10) ле- 
жали внутри единичного круга достаточно, чтобы определители главных миноров матрицы Гурвица, составленной из коэффициентов $u_{0} \ldots u_{4}$ были положительными. Второй способ связан с прямым вычислением корней характеристического уравнения (10).

Решения уравнения (10) имеют следующий вид:

$$
\begin{aligned}
& \lambda_{1}=\frac{1}{2}\left(-\frac{q_{1}}{2}+y_{6}-\sqrt{y_{7}+y_{8}}\right), \\
& \lambda_{2}=\frac{1}{2}\left(-\frac{q_{1}}{2}+y_{6}+\sqrt{y_{7}+y_{8}}\right), \\
& \lambda_{3}=\frac{1}{2}\left(-\frac{q_{1}}{2}-y_{6}-\sqrt{y_{7}-y_{8}}\right), \\
& \lambda_{4}=\frac{1}{2}\left(-\frac{q_{1}}{2}-y_{6}+\sqrt{y_{7}-y_{8}}\right),
\end{aligned}
$$

где $y_{1}=12+q_{2}^{2}-3 q_{1} q_{3}$,

$$
\begin{gathered}
y_{2}=27 q_{1}^{2}-72 q_{2}+2 q_{2}^{3}-9 q_{1} q_{2} q_{3}+27 q_{3}^{2}, \\
y_{3}=-q_{1}^{3}+4 q_{1} q_{2}-8 q_{3}, \quad y_{4}=\frac{q_{1}^{2}}{4}-\frac{2 q_{2}}{3}, \\
y_{5}=\frac{\sqrt[3]{2} y_{1}}{3 \sqrt[3]{y_{2}+\sqrt{-4 y_{1}^{3}+y_{2}^{2}}}}+\frac{\sqrt[3]{y_{2}+\sqrt{-4 y_{1}^{3}+y_{2}^{2}}}}{\sqrt[3]{32}}, \\
y_{6}=\sqrt{y_{4}+y_{5}}, \quad y_{7}=2 y_{4}-y_{5}, \quad y_{8}=\frac{y_{3}}{4 \sqrt{y_{4}+y_{5}}} .
\end{gathered}
$$

Учитывая (6) и (11)-(14) для устойчивости системы необходимо выполнение неравенства $\max _{i}\left|\lambda_{i}\right|<1$. Имея ввиду функциональную зависимость параметров характеристического уравнения от амплитуды, частоты и жёсткости пружины построим зоны устойчивости в пространстве параметров.

\section{ПОСТРОЕНИЕ ЗОН УСТОЙЧИВОСТИ И АНАЛИЗ РЕЗУЛЬТАТОВ}

Границами зоны устойчивости являются линии в пространстве параметров, соответствующие значениям максимального собственного числа равного единице. Для построения этих областей последовательно зафиксируем значения жёсткости пружины на уровнях $k=5,10,20,50,60,95$. Результаты получены численно, с помощью пакета Wolfram Mathematica.
Из рис. 4 видно, что геометрия зон устойчивости претерпевает изменения, соответствующие уширению второй зоны, а также сдвигу первой вправо по оси $A$. Заметим, также, что полученные результаты схожи с графиками, полученными в [16].

На рис. 5 показаны зоны устойчивости в пространстве безразмерных параметров. Для построения этих областей, как и ранее, последовательно фиксировались значения жёсткостипружинынауровнях $k=5,10,20,50,80,100$.

Из рис. 5 видно, что геометрия зон устойчивости претерпевает малые изменения, соответствующие уширению нижней зоны и сдвигу верхней вниз по оси $\beta$. Заметим, также, что полученные результаты схожи с графиками, полученными в [21].

Чтобы проиллюстрировать эволюцию изменения зон устойчивости в зависимости от жёсткости пружины, возьмём нижнюю границу первой зоны устойчивости в пространстве безразмерных параметров.

Параметрам, удовлетворяющим неравенству (6), соответствуют почти периодические колебания [22] маятников относительно верхнего положения. Для иллюстрации динамики системы, приведём фазовые портреты и зависимости фазовых координат (размерных) от времени для значений параметров системы: $l=1 \mathrm{M}, k=95 \mathrm{H} / \mathrm{M}, a=2 \mathrm{M}, \omega=10$ Гц, $\varphi_{1}(0)=0.01, \quad \dot{\varphi}_{1}(0)=-0.01, \quad \varphi_{2}=-0.03$, $\dot{\varphi}_{2}=0.02$. (рис. 7).

В заключении отметим, что полученные движения соответствуют почти периодическим функциям: спектры полученных решений имеют две ярко выраженные гармоники (рис. 8).

\section{ПЕРИОДИЧЕСКИЕ РЕЖИМЫ}

Рассмотрим поведение движения маятника при параметрах, лежащих на границах области устойчивости, что возвращаясь к характеристическому уравнению матрицы монодромии (9) относится к трём случаям: когда $q_{1}=-4, q_{1}=0, q_{1}=4$. Тогда мультипликаторы будут принимать следующие значения: $\lambda_{1}=\lambda_{2}=\lambda_{3}=\lambda_{4}=1, \lambda_{1}=\lambda_{2}=-1 ; \lambda_{3}=\lambda_{4}=1$ и $\lambda_{1}=\lambda_{2}=\lambda_{3}=\lambda_{4}=-1$ соответственно. 
Стабилизация системы связанных обратных маятников вертикальнылми осцилляцчиями

a) $k=5$

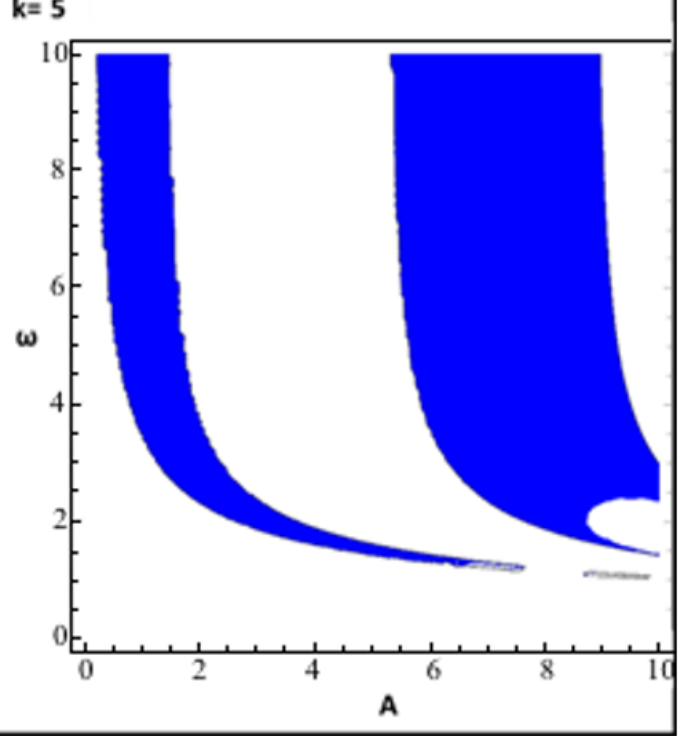

B) $k=20$

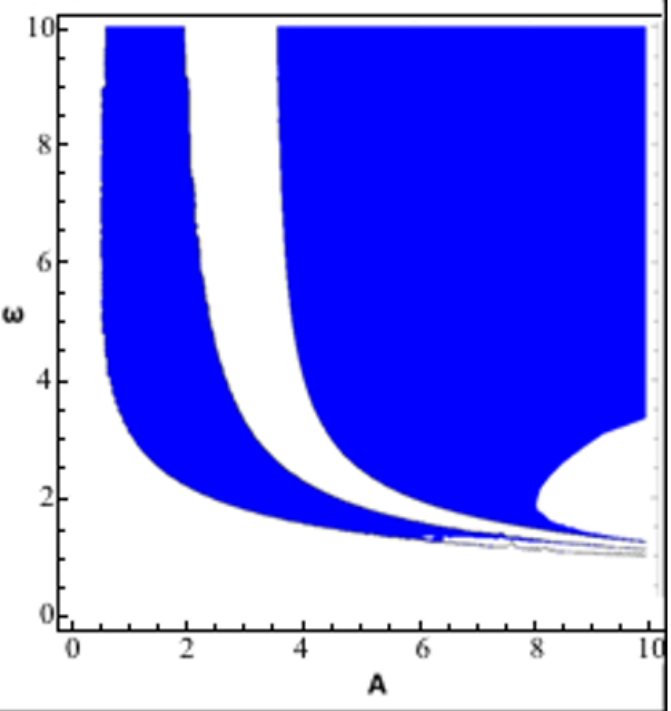

д)

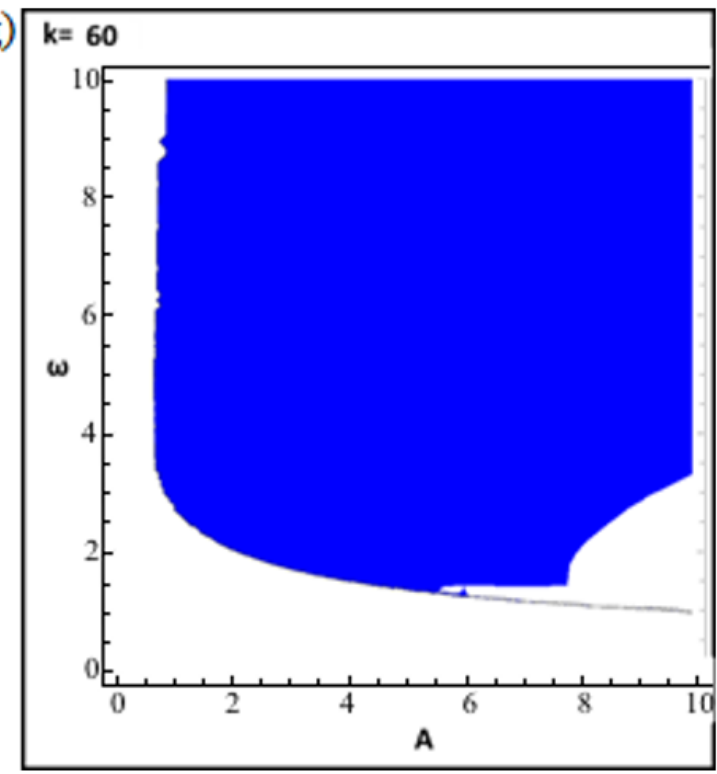

6) $k=10$

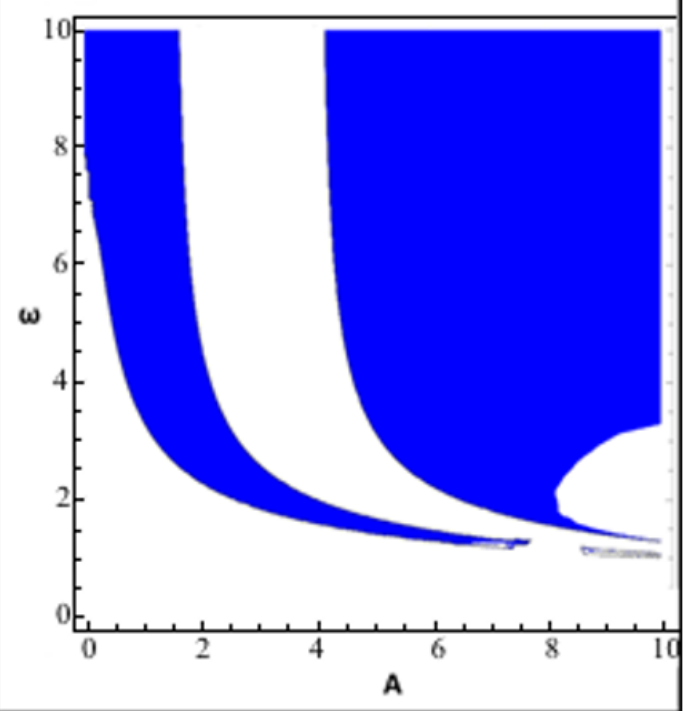

r) $k=50$

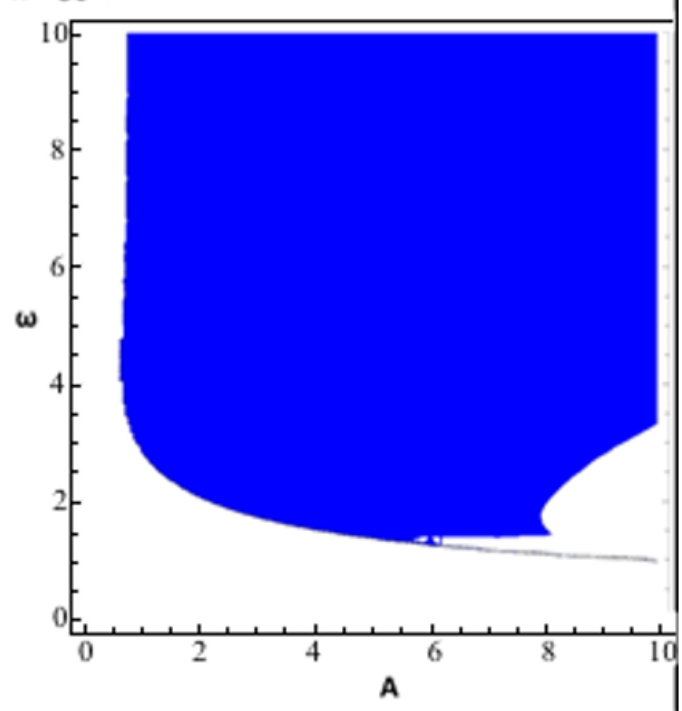

e) $k=95$

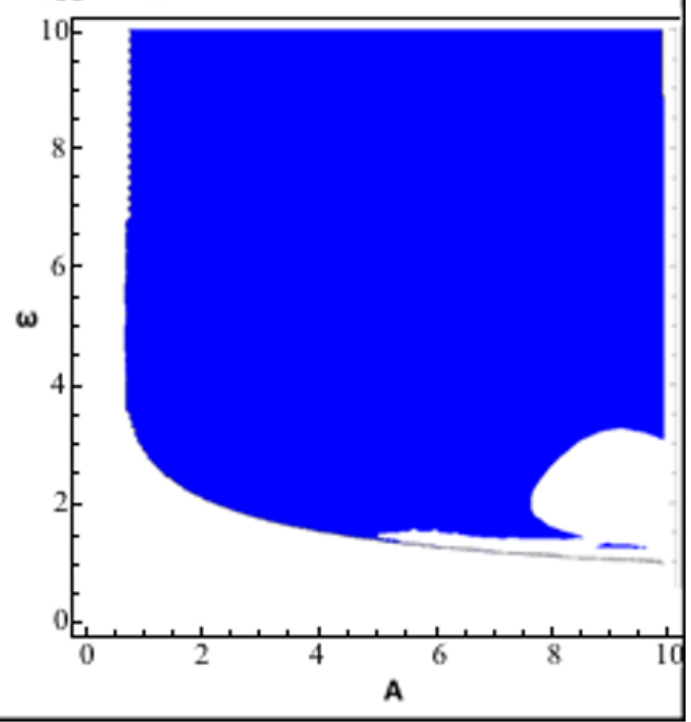

A

Рис. 4. Грабики областей устойчивости при различных жёсткостях пружины $k$ 
a)

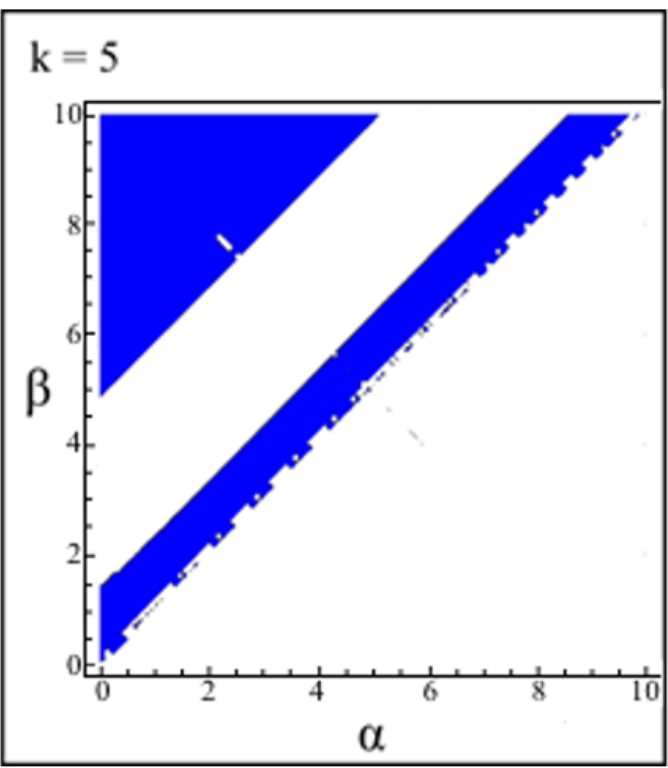

в)

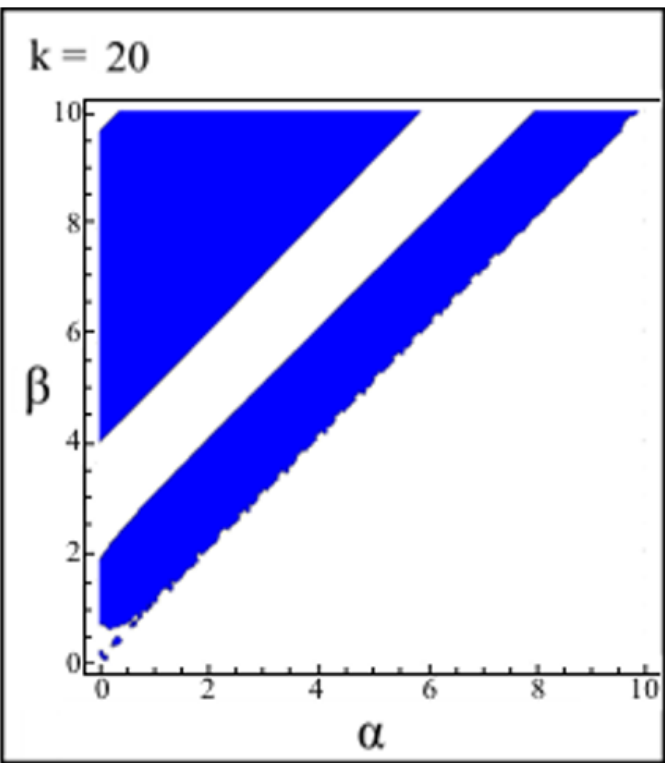

д)

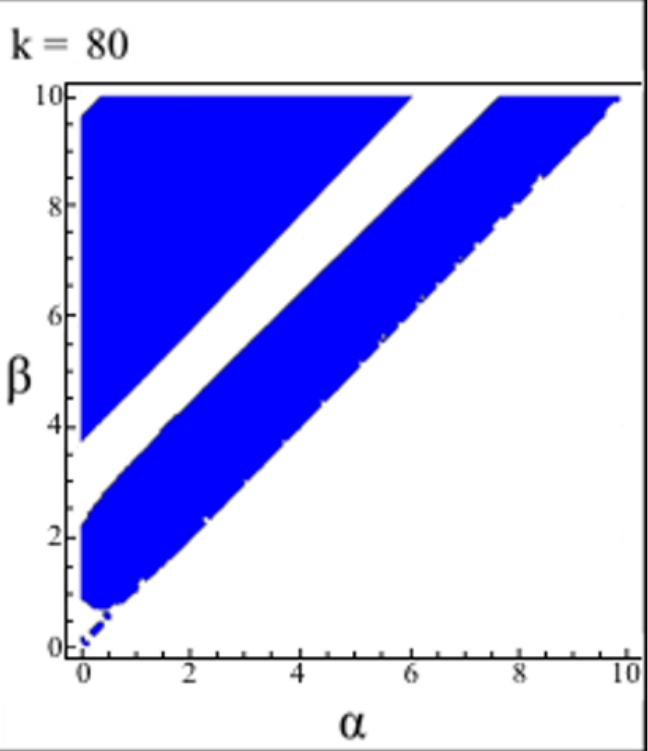

б)

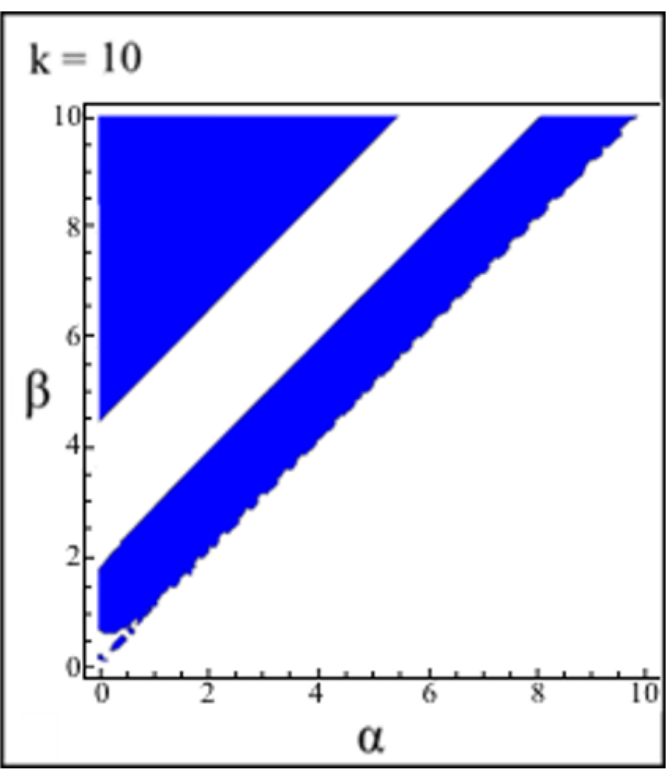

г)

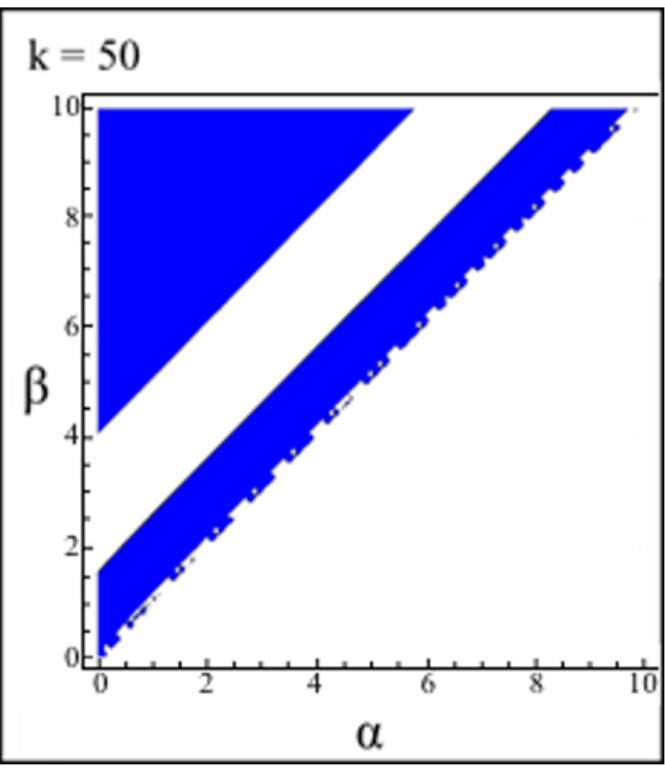

e)

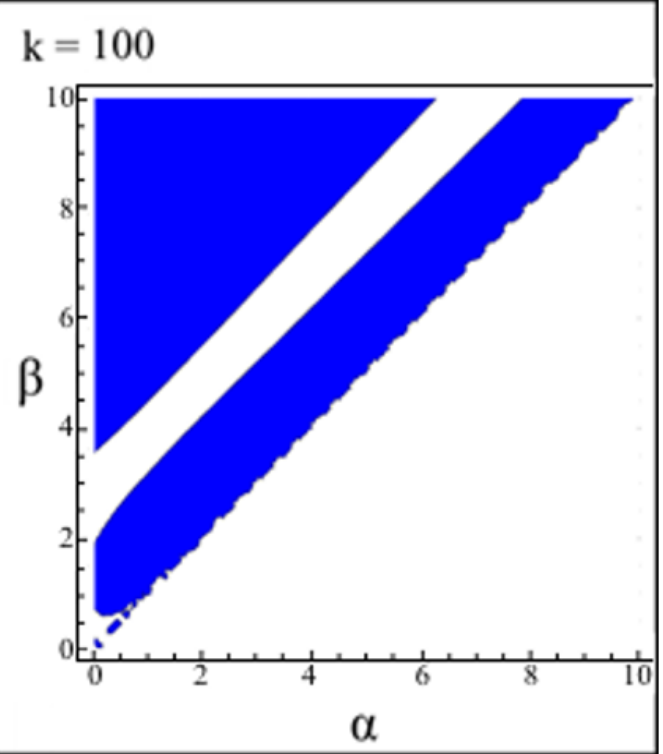

Рис. 5. Графики областей устойчивости при различных жёсткостях пружины $k$ 


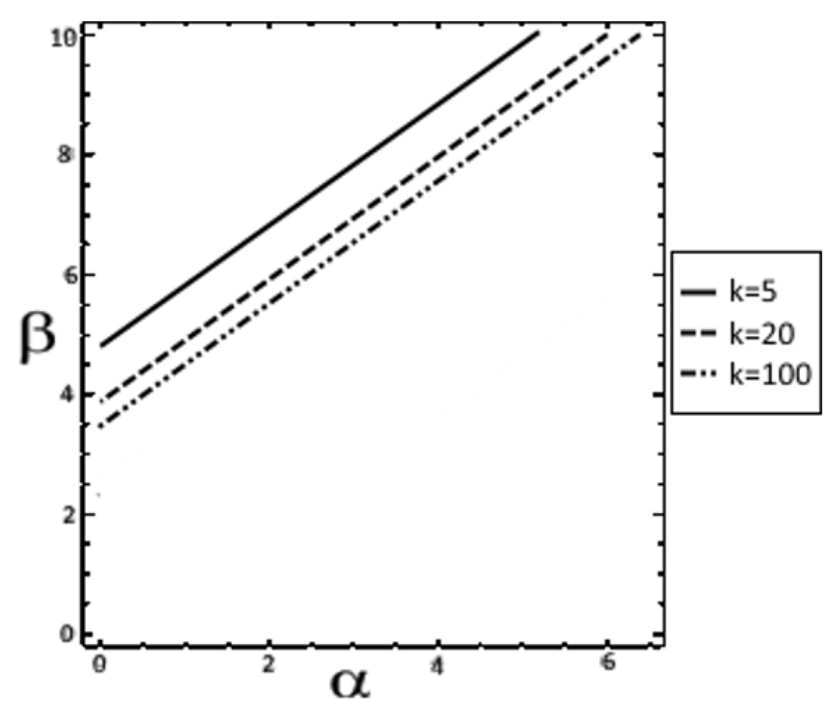

Рис. 6. Верхняя и нижняя границы областей устойчивости при различных $k$

Если $\lambda_{1}=\lambda_{2}=\lambda_{3}=\lambda_{4}=1$, то соответствующее нормальное решение будет удовлетворять равенству $Z(t+2 \pi)=Z(t)$, следовательно, уравнение (2) имеет периодическое решение, период которого совпадает с периодом его коэффициентов $T_{1}=\frac{2 \pi}{\omega}$.

Если $\lambda_{1}=\lambda_{2}=-1 ; \lambda_{3}=\lambda_{4}=1$ или $\lambda_{1}=\lambda_{2}=$ $=\lambda_{3}=\lambda_{4}=-1$,то соответствующее нормальное решение будет удовлетворять равенству $Z(t+2 \pi)=-Z(t)$, а еще через один период $Z(t+4 \pi)=-Z(t+\pi)=Z(t), \quad$ следовательно, уравнение (2) имеет периодические решения, период которых $T_{2,3}=\frac{4 \pi}{\omega}[23]$.

Таким образом, периодические режимы будут иметь место, если будет выполнено одно из соотношений:

$$
\begin{gathered}
q_{1}=4, \text { для периода колебаний } T_{1} \\
q_{1}=-4 \text { или } q_{1}=0, \text { для } \\
\text { периода колебаний } T_{2}
\end{gathered}
$$

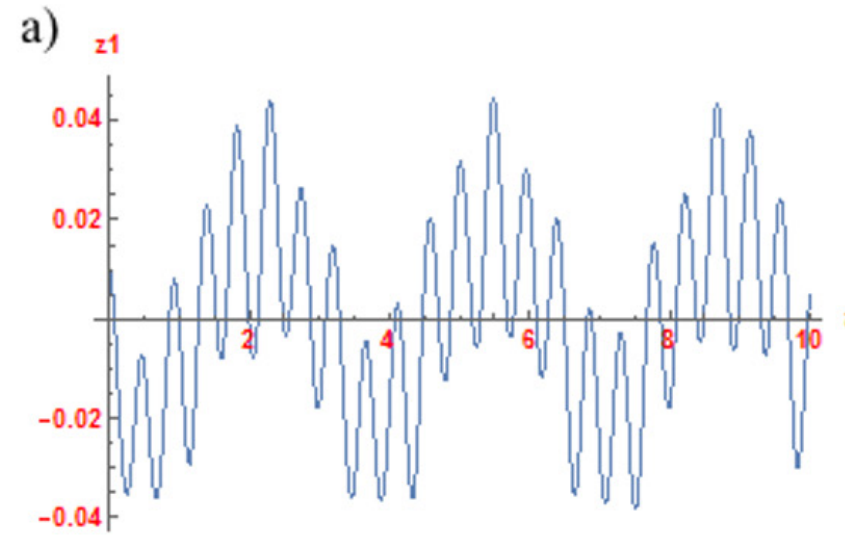

б) 23
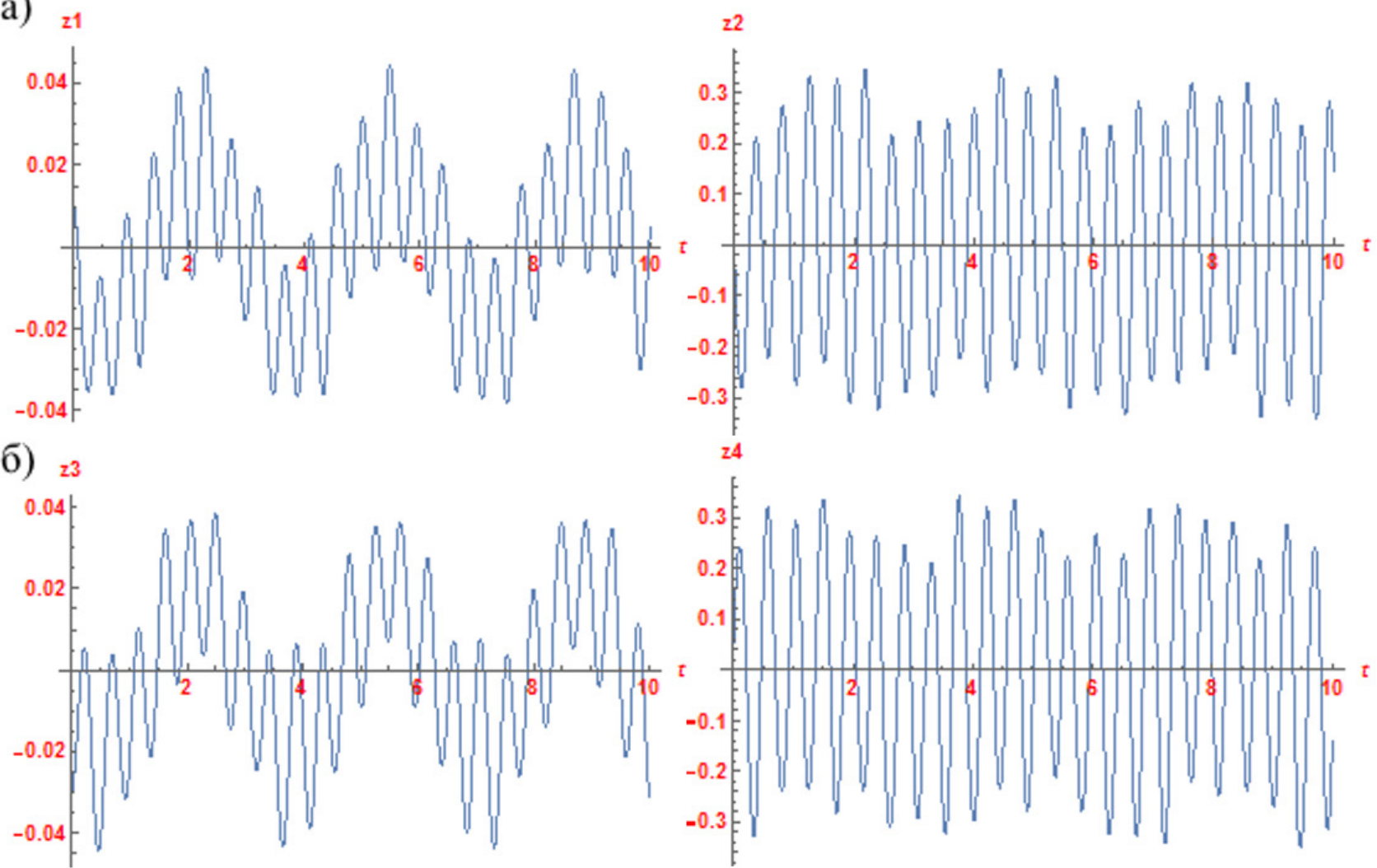

B)

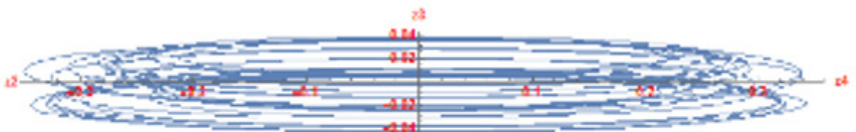

Рис. 7. а) Графики характеристик движения первого маятника б) Грабики характеристик движения второго маятника в) Фазовые плоскости 

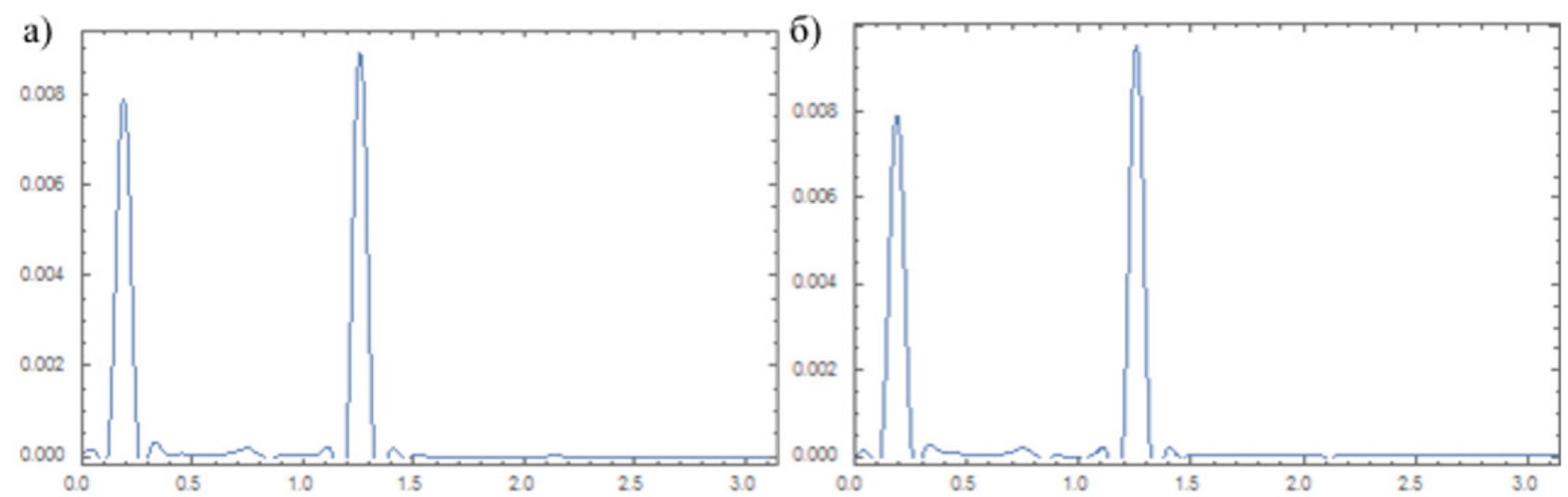

Рис. 8. Спектры решений: слева $z_{1}$, справа $z_{3}$

Это необходимые, но не достаточные условия, поскольку не для любых начальных значений при заданном управлении с параметрами, удовлетворяющими одному из указанных равенств, будут существовать периодические решения.

Отметим, что для рассматриваемого здесь класса управлений, начальные условия лежат в I и III четвертях.

Определим следующее начальное условие $\left(\varphi_{10}, \varphi_{20}, \varphi_{30}, \varphi_{40}\right)$, и рассмотрим периодические колебания с периодом $T_{1}$, тогда должно быть справедливо равенство $Z(0+T)=$ $=H Z(0)=Z(0)$, то есть соответствующий вектор является собственным вектором матрицы монодромии, отвечающим единичному собственному значению:

$$
\left(\begin{array}{llll}
h_{11} & h_{12} & h_{13} & h_{14} \\
h_{21} & h_{22} & h_{23} & h_{24} \\
h_{31} & h_{32} & h_{33} & h_{34} \\
h_{41} & h_{42} & h_{43} & h_{44}
\end{array}\right)\left(\begin{array}{l}
\varphi_{10} \\
\varphi_{20} \\
\varphi_{30} \\
\varphi_{40}
\end{array}\right)=\left(\begin{array}{c}
\varphi_{10} \\
\varphi_{20} \\
\varphi_{30} \\
\varphi_{40}
\end{array}\right) .
$$

Следовательно, начальные условия должны удовлетворять условиям:

$$
\begin{aligned}
\varphi_{10} & =\frac{h_{12}}{1-h_{11}} \varphi_{20}+\frac{h_{13}}{1-h_{11}} \varphi_{30}+\frac{h_{14}}{1-h_{11}} \varphi_{40}, \\
\varphi_{20} & =\frac{h_{21}}{1-h_{22}} \varphi_{10}+\frac{h_{23}}{1-h_{22}} \varphi_{30}+\frac{h_{24}}{1-h_{22}} \varphi_{40}, \\
\varphi_{30} & =\frac{h_{31}}{1-h_{33}} \varphi_{10}+\frac{h_{32}}{1-h_{33}} \varphi_{20}+\frac{h_{42}}{1-h_{33}} \varphi_{40}, \\
\varphi_{40} & =\frac{h_{41}}{1-h_{44}} \varphi_{10}+\frac{h_{42}}{1-h_{44}} \varphi_{20}+\frac{h_{43}}{1-h_{44}} \varphi_{30} .
\end{aligned}
$$

Введём новые параметры посредством следующих обозначений: $\frac{h_{12}}{1-h_{11}}=n_{12}$,

$$
\begin{aligned}
\frac{h_{13}}{1-h_{11}}= & n_{13}, \ldots, \frac{h_{43}}{1-h_{44}}=n_{43}, \text { тогда: } \\
\varphi_{20} & =\frac{n_{21}+n_{24} n_{41}}{1-n_{24} n_{42}} \varphi_{10}+\frac{n_{23}+n_{24} n_{43}}{1-n_{24} n_{42}} \varphi_{30}, \\
\varphi_{40} & =\frac{n_{41}+n_{42} n_{21}}{1-n_{42} n_{24}} \varphi_{10}+\frac{n_{43}+n_{42} n_{23}}{1-n_{42} n_{24}} \varphi_{30}
\end{aligned}
$$

т. е. начальные условия, отвечающие периодическим решениям, лежат на плоскости

$$
\begin{aligned}
& \dot{\varphi}_{20}=K_{11} \varphi_{10}+K_{12} \varphi_{30}, \\
& \dot{\varphi}_{40}=K_{21} \varphi_{10}+K_{22} \varphi_{30},
\end{aligned}
$$

где коэффициенты имеют следующие значения:

$$
\begin{aligned}
& K_{11}=\frac{h_{21}\left(1-h_{44}\right)+h_{24} h_{41}}{\left(1-h_{22}\right)\left(1-h_{44}\right)-h_{24} h_{42}}, \\
& K_{12}=\frac{h_{23}\left(1-h_{44}\right)+h_{24} h_{43}}{\left(1-h_{22}\right)\left(1-h_{44}\right)-h_{24} h_{42}}, \\
& K_{21}=\frac{h_{41}\left(1-h_{22}\right)+h_{42} h_{21}}{\left(1-h_{44}\right)\left(1-h_{22}\right)-h_{42} h_{24}}, \\
& K_{22}=\frac{h_{43}\left(1-h_{22}\right)+h_{42} h_{23}}{\left(1-h_{44}\right)\left(1-h_{22}\right)-h_{42} h_{24}} .
\end{aligned}
$$

Если для начальных условий $\left(\varphi_{10}, \varphi_{20}, \varphi_{30}, \varphi_{40}\right)$ можно найти пару параметров $A$ и $\omega$, лежащих на границе области устойчивости, удовлетворяющую равенству (17), то эта пара единственна. Обратное так же верно.

Аналогично определяется плоскость, соответствующая начальным условиям, отвечающим периодическим решениям с периодом $T_{2}$. 


\section{ЗАКЛЮЧЕНИЕ}

В работе рассматривалась динамика системы, состоящей из неустойчивых связанных маятников, находящихся под воздействием сил инерции, обусловленных вертикальными осцилляциями нижней точки крепления. Было показано, что наличие жёсткой упругой связи между маятниками коренным образом меняет динамику системы. Были построены зоны устойчивости в пространстве естественных и безразмерных параметров, а также, исследована эволюция зон устойчивости в зависимости от жёсткости пружины. Установлено наличие неустойчивых периодических режимов на границах зон устойчивости.

Работа выполнена при финансовой поддержке РФФИ (проекты 17-01-00251 и 19-0800158)

\section{СПИСОК ЛИТЕРАТУРЫ}

1. Андронов А. А., Витт А. А., Хайкин С. Э. Теория колебаний. - М. : Наука, 1981. - 568 с.

2. Баутин Н. Н. О числе предельных циклов, появляющихся при изменении коэффициентов из состояния равновесия типа фокуса или центра // Математический сборник. 1952. - 30(72). - C. 181-196.

3. Трубецков Д. И., Рожнев А. Г. Линейные колебания и волны. - М. : Издательство Физико-математической литературы, 2001. - 416 с.

4. Магнус К. Колебания: Введение в исследование колебательных систем. Пер. с нем. М. : Мир, 1982. 304 с.

5. Осипов Г. В. Синхронизация в неоднородных сетях осцилляторов. - Нижний Новгород, 2014. - 135 c.

6. Колмогоров А. Н. О сохранении условно периодических движений при малом изменении функции Гамильтона // Доклады АН CССР. - 1954. - 98(4). - С. 527-530.

7. Бутиков Е. И. Стабилизация перевернутого маятника (60 лет маятнику Капицы), Компьютерные инструменты в образовании. - 2010. - 5. - 3951.

8. Stephenson A. On an induced stability // Phil. Mag. - 1908. - 15(233).
9. Капица П. Л. Маятник с вибрирующим подвесом / П. Л. Капица // УФН 44. - 1951. C. 7-20.

10. Капища П. Л. Динамическая устойчивость маятника при колеблющейся точке подвеса / П. Л. Капица //ЖЭТФ 21. - 1951. С. 588-597.

11. Нелепин Р. А. Методы исследования нелинейных систем автоматического управления / Под ред. Р.А. Нелепина. - М. : Наука,1979. - 447 с.

12. Красносельский М. А. Системы с гистерезисом / М. А. Красносельский, А. В. Покровский - М. : Наука, 1983. - 271 с.

13. Плисс В. А. Нелокальные проблемы теории колебаний / В. А. Плисс. - М. : Наука, 1964. - 367 c.

14. Меркин Д. Р. Введение в теорию устойчивости движения: Учеб. Пособие для вузов / Д. Р. Меркин - 3-е изд., перераб. и доп. - М. : Наука, 1987. - 304 с.

15. Матвеев М. Г., Семёнов М. Е., Шевлякова Д. В., Канищева О. И. Зоны устойчивости и периодические решения перевернутого маятника с гистерезисным управлением // Мехатроника, Автоматизация, Управление. 2012. - 11. - C. 8-14.

16. Семенов М. Е., Хатиळ 3., Решетова О. О., Демчук А. А., Мелешенко П. А. Модель динамики обратного маятника с гистерезисным управлением // Вестник Воронеж. гос. ун-та. Сер. Физика. Математика. - 2016. 4. - C. 165-177.

17. Семёнов М. Е., Матвеев М. Г., Лебедев Г. Н., Соловьёв А. М. Стабилизация обратного гибкого маятника с гистерезисными свойствами // Мехатроника, Автоматизация, Управление. - 2017. - 8. - С. 516-525.

18. Семенов М. Е., Соловьев А. М., Попов М. А. Стабилизация неустойчивых объектов: связанные осцилляторы // Труды МАИ. 2017. - C. 93.

19. Solovyov A. M., Semenov M. E., Meleshenko P. A., Reshetova O. O., Popov M. A., Kabulova E. G. Hysteretic nonlinearity and unbounded solutions in oscillating systems // Procedia Engineering. - 2017. - 201 P. 549-555.

20. Semenov M. E., Solovev A. M., Popov M. A, Meleshenko P. A. Coupled inverted pendulums: 
stabilization problem // Archive Of Applied Mechanics. - 2018. - 88. - C. 517-524.

21. Неймарк Ю. И., Коган Н. Я., Савельев В. П. Динамические модели теории управления. - М. : Наука, 1985. - 400 с.

22. Красносельский M. А. Нелинейные почти периодические колебания / М. А. Крас-

Семенов Михаил Евгеньевич - д-р физ.-мат. наук, профессор, ведущий научный сотрудник ФГБУН Федеральный исследовательский центр «Единая геофизическая служба Российской академии наук», Обнинск, Россия; профессор кафедры цифровых технологий, Воронежский государственный университет, Воронеж, Россия.

E-mail: mkl150@mail.ru

Попов Михаил Александрович - аспирант кафедры прикладной математики и механики, Воронежский государственный технический университет.

E-mail: soeltic@gmail.com. носельский, В. Ш. Бурд, Ю. С. Колесов. - М. : Наука, 1970. - 304 с.

23. Мирошник И. В. Теория автоматического управления. Нелинейные и оптимальные системы. - СПб. : Питер, 2006. - 272 с.

Semenov Mikhail E. - Geophysical Survey of Russia Academy of Sciences, Obninsk, Russia; Digital Technologies Department, Voronezh State University, Voronezh, Russia; Prof., senior professor.

E-mail: mkl150@mail.ru

Popov Mikhail A. - Voronezh State Technical University, Applied Mathematics and Mechanics Department.

E-mail: soeltic@gmail.com 